Pacific Journal of Mathematics

A NUMERICAL RANGE FOR TWO LINEAR OPERATORS 


\title{
A NUMERICAL RANGE FOR TWO LINEAR OPERATORS
}

\author{
Charles F. Amelin
}

\begin{abstract}
A numerical range for two closed, linear operators is defined for the purpose of obtaining some new results on the stability of index of a Fredholm operator perturbed by a bounded or relatively bounded operator.
\end{abstract}

o. Introduction. One of the objects of the present paper is to study the eigenvalue problem $T x=\lambda A x$ by means of a two (linear) operator or "bioperative" numerical range. We recall that Toeplitz [30] defined the numerical range for matrices in 1918. Then Wintner [31] in 1930 and Stone [27], [28] in 1930 and 1932 discussed the relationship between the convex hull of the spectrum of a bounded linear operator on a Hilbert space and its numerical range. In 1943, J. Dieudonné [4] and S. M. Nikol'skii [24] laid the groundwork which would later help to show that the index of a bounded semi-Fredholm operator is stable under perturbation by a bounded linear operator of sufficiently small norm. This result was established in 1951 for bounded operators on a Hilbert space by F.V.Atkinson [2] and (independently) I. C. Gohberg [9], [10], and [11]. The following year, M. G. Krein and M. A. Krasnosel'skii [20], B. Sz.-Nagy [29], and I. C. Gohberg [12] generalized these results to unbounded closed linear operators. M. G. Krein and M. A. Krasnosel'skii also established the semi-stability of the nullity and deficiency. To understand the foundations and historical development of the whole theory, the reader is referred to the comprehensive article of Gohberg and Krein [13] which appeared in 1957.

Among the many innovations appearing in the 1958 paper of $\mathrm{T}$. Kato [18] was the concept of the "lower bound" (now called the "minimum modulus") of a linear operator $A$ defined on a Banach space. The main reason for defining the minimum modulus of $A$ was to obtain as small a disc about the origin as possible so that ind $(T-\lambda A)=\operatorname{ind}(A)$ for all $\lambda$ outside that disc, $A$ being semi-Fredholm and $T$ being bounded or relatively bounded with respect to $A$. In this paper we introduce a bioperative numerical range which will improve that result for Fredholm operators defined on a Hilbert space. The improvement is a consequence of the fact that the bioperative numerical range for Fredholm $A$ and relatively bounded $T$ defined on a Hilbert space is always contained in the aforementioned disc and that the index of $T-\lambda A$ remains constant if $\lambda$ is not in the closure of that bioperative numerical range.

For another use of the bioperative numerical range, we recall 
that W.Givens [8] showed that $\operatorname{co} \sigma(T)=\bigcap\left\{\overline{W\left(S T S^{-1}\right)}: S\right.$ is invertible $\}$ if $\operatorname{dim}(H)<\infty$. S. Hildebrandt [17] extended this theorem in 1966 to bounded linear operators defined on infinite dimensional Hilbert spaces $H$. In 1968, J. G. Stampfli and J. P. Williams [26] defined an "essential" numerical range $W_{e s s}(T)$ for $T \in \mathscr{B}(H)$. It was shown that $W_{e s s}(T)=\bigcap\{\overline{W(T+K}): K$ is compact $\}$ and co $\sigma_{e s s}(T) \subset W_{e s s}(T)$. According to a result of Stampfli and Williams [26], if $T$ is normal then co $\sigma_{e s s}(T)=W_{e s s}(T)$, but equality does not hold in general. In paragraph 2 of this paper we obtain the convex hull of the essential spectrum of a bounded linear operator defined on a separable Hilbert space in terms of intersections of appropriate bioperative numerical ranges.

In paragraph 3 , we indicate how to extend some of the results of the first paragraph to Banach and Frechét spaces. We give two examples in paragraph 4. Any one of the texts Kato [19], Goldberg [14], or Schechter [25] would be a suitable introductory reference to the elementary definitions and basic theory assumed here.

I would like to thank Robert T. Moore of the University of Washington for suggesting a problem in generalized spectral theory which led to this paper, and Seymour Goldberg and David Lay of the University of Maryland for their suggestions.

\section{Stability of index theorems in Hilbert space.}

Definition 1.1. Let $H$ and $K$ be Hilbert spaces and $T, A \in$ $\mathscr{C}(H, K)$. We define $W(T, A)=\{(T x, A x):\|A x\|=1, x \in \mathscr{D}(A) \cap \mathscr{D}(T)\}$.

Clearly, if $H=K, A$ is the identity, and $T \in \mathscr{B}(H)$, then $W(T, A)$ is the Toeplitz numerical range $W(T)=\{(T x, x):\|x\|=1\}$. If $A^{-1} \in \mathscr{B}(H)$, then $W\left(T A^{-1}\right)=W(T, A)$.

Lemma 1.2. $W(T, A)$ is a convex set.

Proof. We may assume that there exist two distinct points $\lambda_{1}, \lambda_{2} \in W(T, A)$ since the lemma is trivial otherwise. I.e., there exist $x_{1}, x_{2} \in \mathscr{D}(A) \cap \mathscr{D}(T)$ such that $\left(T x_{1}, A x_{1}\right)=\lambda_{1}\left\|A x_{1}\right\|=1$ and $\left(T x_{2}, A x_{2}\right)=\lambda_{2},\left\|A x_{2}\right\|=1$. Since $\mathscr{D}(A) \cap \mathscr{D}(T)$ is a subspace of $H$ we may consider the binary forms $C_{i}: C \times C \rightarrow C, i=1,2$ defined by

$$
\begin{array}{ll}
C_{1}\left(\alpha_{1}, \alpha_{2}\right)=\left(T\left(\alpha_{1} x_{1}+\alpha_{2} x_{2}\right),\right. & \left.A\left(\alpha_{1} x_{1}+\alpha_{2} x_{2}\right)\right) \\
C_{2}\left(\alpha_{1}, \alpha_{2}\right)=\left(A\left(\alpha_{1} x_{1}+\alpha_{2} x_{2}\right),\right. & \left.A\left(\alpha_{1} x_{1}+\alpha_{2} x_{2}\right)\right) .
\end{array}
$$

We must show that $C_{1}$ assumes every value on the line segment joining $\lambda_{1}$ and $\lambda_{2}$ while $C_{2}=1$. If we let 


$$
C\left(\alpha_{1}, \alpha_{2}\right)=\frac{C_{1}-\lambda_{2} C_{2}}{\lambda_{1}-\lambda_{2}}=\alpha_{1} \bar{\alpha}_{1}+a_{12} \bar{\alpha}_{1} \alpha_{2}+a_{21} \alpha_{1} \bar{\alpha}_{2}
$$

where

$$
\left(\lambda_{1}-\lambda_{2}\right) a_{12}=\left(T x_{2}, A x_{1}\right)-\lambda_{2}\left(A x_{2}, A x_{1}\right)
$$

and $\left(\lambda_{1}-\lambda_{2}\right) a_{21}=\left(T x_{1}, A x_{2}\right)-\lambda_{2}\left(A x_{1}, A x_{2}\right)$, then it suffices to show that the form $C$ takes on every real value from 0 to 1 , while $C_{2}=1$. Choose $\beta$ of modulus 1 so that $\left(a_{12}-\bar{a}_{21}\right) \beta$ is real and

$$
\operatorname{Re}\left(A x_{1}, A x_{2}\right) \bar{\beta} \geqq 0 \text {. }
$$

If $\alpha_{1}=u$ and $\alpha_{2}=\beta v$ where $u$ and $v$ are real variables, then $C=$ $u^{2}+r u v$ and $C_{2}=u^{2}+2 s u v+v^{2}$ where $r=\beta a_{12}+\bar{\beta} a_{21}$ is real and $s \geqq 0$. Solving for $C_{2}(u, \beta v)=1$ we obtain $v=-s u+\sqrt{1+\left(s^{2}-1\right) u^{2}}$ which is real valued for $u \in[-1,+1]$. Since $v$ is now a function of $u$, we can let $C_{0}(u)=C(u, \beta v)=u^{2}(1-r s)+r u \sqrt{1+\left(s^{2}-1\right) u^{2}}$. For $u \in[0,1], C_{0}(u)$ is evidently a continuous real valued function with $C_{0}(0)=0, C_{0}(1)=1$ so that $C_{0}(u)$ assumes all values between 0 and 1.

This proof was adapted from the proof given by Stone [28], p.131. Certain other facts about the bioperative numerical range are easy to verify; e.g., $W(\alpha T+\beta S, A) \subset \alpha W(T, A)+\beta W(S, A)$ where we set $\mathscr{D}(\alpha T+\beta S)=\mathscr{D}(T) \cap \mathscr{D}(S)$. For another fact, let $T, A \in \mathscr{B}(H)$ and $T^{*}, A^{*}$ denote the Hilbert space adjoints of $T$ and $A$. Then $W(T, A)$ lies on the line $y=x \tan \left(\theta_{0}\right)$ if $A^{*} T=e^{2 i \theta_{0}} T^{*} A$. In particular, if $T$ and $A$ are self-adjoint and commute, then $W(T, A)$ is real.

In what follows if $A \in \mathscr{C}(H, K)$, we let $P_{A}$ denote the projection $P_{A}: H \rightarrow N(A)$ onto the orthogonal complement of the nullspace of $A$, and we denote the class of semi-Fredholm operators with finite nullity by $\Phi_{+}$. We recall that the minimum modulus of $A \in \mathscr{C}(H, K)$, is the greatest number $\gamma$ which satisfies the inequality

$$
\gamma \operatorname{dist}(x, N(A)) \leqq\|A x\|
$$

for every $x \in \mathscr{Z}(A)$. Thus

$$
\gamma(A)=\inf \left\{\frac{\|A x\|}{\operatorname{dist}(x, N(A))}: x \in D(A)\right\}
$$

and

$$
\gamma\left(\left.A\right|_{x(A)^{2}}\right)=\inf \left\{\frac{\|A x\|}{\left\|P_{A} x\right\|}: x \in D(A)\right\}
$$

(where $0 / 0=\infty$ ). If $x \in H$ and $x=x_{1}+n \in N(A) \oplus N(A)$, then 
$\|x\|^{2}=\left\|x_{1}\right\|^{2}+\|n\|^{2}$ and $\operatorname{dist}(x, N(A))=\left\|x_{1}\right\|=\left\|P_{A} x\right\|$. In other words, if $H$ is a Hilbert space and $A \in \mathscr{C}(H, K)$, then

$$
\gamma(A)=\gamma\left(\left.A\right|_{N(A) \perp}\right) \text {. }
$$

THEOREM 1.3. Let $T, A \in \mathscr{C}(H, K)$ with $\mathscr{D}(T) \supset \mathscr{D}(A)$. If $A \in \Phi_{+}$ and $\lambda \notin \overline{W\left(T P_{A}, A\right)}, T-\lambda A \in \Phi_{+}$, then $\operatorname{ind}(T-\lambda A)=\operatorname{ind}(A), \operatorname{nul}(T-\lambda A) \leqq$ $\operatorname{nul}(A)$, and $\operatorname{def}(T-\lambda A) \leqq \operatorname{def}(A)$.

Proof. Since $T, A \in \mathscr{C}(H, K)$ and $\mathscr{D}(T) \supset \mathscr{D}(A)$ there exist nonnegative constants $a, b$ such that $\|T x\| \leqq a\|x\|+b\|A x\|$ for every $x \in \mathscr{D}(A)$. In particular, $\left\|T P_{A} x\right\| \leqq a\left\|P_{A} x\right\|+b\|A x\|$ for every $x \in \mathscr{D}(A)$. We recall that Kato [18] has proven that if

$$
|\xi|>\frac{a}{\gamma(A)}+b
$$

then $(T-\xi A) P_{A}$ is semi-Fredholm and ind $\left((T-\xi A) P_{A}\right)=$ ind $(A)$. But if $\lambda \notin \overline{W\left(T P_{A}, A\right)}$, we can find a $\delta>0$ such that $(*) \delta\|A x\| \leqq$ $\left\|(T-\lambda A) P_{A} x\right\|$ for every $x \in \mathscr{D}(A)=\mathscr{D}(T-\lambda A)=\mathscr{D}\left((T-\lambda A) P_{A}\right)$. (Apply the Schwarz inequality and the fact that $\|A x\|=1$, to the inequality $\left.\delta \leqq 1\left(T P_{A} x, A x\right)-\lambda \mid\right)$. The starred inequality implies that $(T-\lambda A) P_{A}$ is closed. By the same inequality $\operatorname{nul}\left((T-\lambda A) P_{A}\right) \leqq \operatorname{nul}(A)$ so that the dimensions $\operatorname{nul}\left((T-\lambda A) P_{A}\right)$ and $\operatorname{nul}(A)$ are equal by the definition of $P_{A}$. (Notice $\left.A=A P_{A}\right)$. Furthermore, since $H$ is a Hilbert space, $\gamma(A)$ equals $\gamma\left(\left.A\right|_{N(A) \perp}\right)$ and thus $\gamma(A)\left\|P_{A} x\right\| \leqq\|A x\|$ so that the range of $(T-\lambda A) P_{A}$ is closed (by the starred inequality); i.e., $(T-\lambda A) P_{A}$ is semi-Fredholm with finite nullity for every $\lambda \notin \overline{W\left(T P_{A}, A\right)}$. Since $\overline{W\left(T P_{A}, A\right)}$ is a convex, subset of the complex plane, its complement is connected. But this implies by the asserted result of Kato et al., that

$$
\text { ind }\left((T-\lambda A) P_{A}\right)=\text { ind }(A)
$$

for every $\lambda \notin \overline{W\left(T P_{A}, A\right)}$ because $\phi(\lambda)=$ ind $\left((T-\lambda A) P_{A}\right)$ is a constant function on connected subsets of the plane for which $(T-\lambda A) P_{A}$ is semi-Fredholm. $T-\lambda A$ must have nullity smaller than that of $\operatorname{nul}(A)=\operatorname{nul}\left(P_{A}\right)$, because $(T-\lambda A) P_{A}$ is one-to-one on $N(A)^{\perp}$ and equals $T-\lambda A$ on $N(A)^{\perp}$. In fact, suppose there exists an orthonormal set $\left\{x_{i}: i=1, \cdots, n+1\right\} \subset \mathscr{D}(T-\lambda A)$ with $n=\operatorname{nul}(A),(T-\lambda A) x_{i}=0$. Write $x_{i}=p_{i}+q_{i} \in N(A)^{\perp} \oplus N(A)$. Since $T-\lambda A$ is one-to-one on $N(A)^{\perp}, q_{i} \neq 0$ for every $i$. At most $n$ of the $q_{i}$ are linearly independent so $q_{n+1}=\sum_{i=1}^{n} \beta_{i} q_{i}$. Since

$$
(T-\lambda A) p_{i}=-(T-\lambda A) q_{i},(T-\lambda A) p_{n+1}=\sum_{i=1}^{n} \beta_{i}(T-\lambda A) p_{i}
$$

and thus $p_{n+1}=\sum_{i=1}^{n} \beta_{i} p_{i}$ by the linearity of the one-to-one map 
$T-\lambda A$. The resulting fact, $x_{n+1}=\sum_{i=1}^{n} \beta_{i} x_{i}$ is a contradiction so $\operatorname{nul}(T-\lambda A) \leqq \operatorname{nul}(A)$.

Further,

$$
(T-\lambda A) \mathscr{D}(A)=(T-\lambda A) N(A)+(T-\lambda A)\left(N(A)^{\prime} \cap \mathscr{D}(A)\right)
$$

is closed being the sum of two closed subspaces one of which is finite dimensional. Since $T-\lambda A$ is thereby semi-Fredholm for every $\lambda \notin \overline{W\left(T P_{A}, A\right)}$,

$$
\operatorname{ind}(T-\lambda A)=\operatorname{ind}(T-\lambda A)+\operatorname{ind}\left(P_{A}\right)=\operatorname{ind}\left((T-\lambda A) P_{A}\right)=\operatorname{ind}(A) .
$$

But then $\operatorname{def}(T-\lambda A) \leqq \operatorname{def}(A)$.

By the Schwarz inequality

$$
\left|\left(T P_{A} x, A x\right)\right| \leqq\left\|T P_{A} x\right\| \leqq a\left\|P_{A} x\right\|+b\|A x\| \leqq \frac{a}{\gamma(A)}+b
$$

if $\|A x\|=1$. We recall that Kato [18] proved that if

$$
|\lambda|>\frac{a}{\gamma(A)}+b
$$

and $A$ is semi-Fredholm, then ind $(T-\lambda A)=$ ind $(A)$. Since we have shown that $\overline{W\left(T P_{A}, A\right)} \subset\{z:|z| \leqq(a / \gamma(A))+b\}$, Theorem 1.3 always gives results at least as good as those previous for $A \in \Phi_{+}$defined on a Hilbert space. The examples in paragraph 4 demonstrate that $W\left(T P_{A}, A\right)$ sometimes gives better results. We note that the stability of index result ind $(T-\lambda A)=$ ind $(A)$ of Theorem 1.3 remains valid for $\lambda \notin \bigcap\left\{\overline{W\left(T P_{A}+K, A\right)}: K\right.$ is compact $\}$ but the semi-stability of the nullity and deficiency need no longer hold.

For the case of semi-Fredholm $A$ with finite deficiency it is possible to define a "dual" bioperative numerical range

$$
W\left(T^{*}, A^{*}\right)^{-}=\left\{\left(A^{*} x, T^{*} x\right):\left\|A^{*} x\right\|=1, x \in \mathscr{D}\left(A^{*}\right) \cap \mathscr{D}\left(T^{*}\right)\right\}
$$

and prove similar results using $W\left(T^{*} P_{A^{*}}, A^{*}\right)^{-}$in place of $W\left(T P_{A}, A\right)$ being careful to hypothesize $\mathscr{D}\left(T^{*}\right) \supset \mathscr{D}\left(A^{*}\right)$. (The small bar denotes complex conjugation.) If $T, A \in \mathscr{B}(H, K)$ and $A$ is Fredholm it is easy to verify the stability results for $\lambda \notin \overline{W\left(T P_{A}, A\right)} \cap \overline{W\left(T^{*} P_{A^{*}}, A^{*}\right)^{-}}$. This may be of interest since both $\overline{W\left(T P_{A}, A\right)}$ and $\overline{W\left(T^{*} P_{A^{*}}, A^{*}\right)^{-}}$are contained in $\{z:|z| \leqq(\|T\| / \gamma(A))\}$.

For simplicity suppose now that $T$ and $A$ are bounded,

$$
r(T, A)=\sup \{|\lambda|: T-\lambda A \text { is not Fredholm }\}
$$

and $|W|(T, A)=\sup \{|\lambda|: \lambda \in W(T, A)\}$. 
We have seen that if $A$ is Fredholm, $r(T, A) \leqq|W|\left(T P_{A}, A\right)$. If $T$ is not Fredholm, then $1 \leqq r(A-T, A)$ so we have just proven the following: If $|W|\left(A-T P_{A}, A\right)<1$ and $A$ is Fredholm, then $T$ is Fredholm.

Theorem 1.3 holds if we replace $W\left(T P_{A}, A\right)$ by $W(T, A)$ since $W\left(T P_{A}, A\right) \subset W(T, A)$. But $W(T, A)$ may be too large. In fact, let $A, T \in \mathscr{B}(H), A$ be Fredholm, and $T(N(A)) \not \subset R(A)^{\perp}$. Then there exists an $n \in N(A)$ and an $x \in H$ such that $(T n, A x) \neq 0$, $\|A x\|=$ $\|A(x+\alpha n)\|=1$ for every $\alpha \in C$ and

$$
(T(x+\alpha n), A(x+\alpha n))=(T x, A x)+\alpha(T n, A x),
$$

so that $W(T, A)=C$. On the other hand, one can verify that the condition $T(N(A)) \subset R(A)^{\lrcorner}$or the condition that $N(A)$ reduces both $T$ and $A$ each guarantee that $W(T, A)=W\left(T P_{A}, A\right)$.

If $A \in \mathscr{C}(H, K)$, then $H=N(A) \oplus N(A)^{\perp}, K=\overline{R(A)} \oplus R(A)^{\perp}$ and $A$ is a one-to-one operator from $N(A)^{\perp}$ onto $R(A) . A^{+}$, the generalized inverse of $A$, has domain $\mathscr{D}\left(A^{+}\right)=R(A) \oplus R(A)^{\perp}$ and is defined to be zero on $R(A)^{\perp}$ and the inverse of the one-to-one operator induced by $A$ on $R(A)$; i.e., $A^{+} A x=P_{A} x, x \in \mathscr{D}(A) . \quad(x \in \mathscr{D}(A) \subset \mathscr{D}(T))$ Since

$$
\left(T P_{A} x, A x\right)=\left(T A^{+} A x, A x\right),
$$

the following reduction of the bioperative numerical range to the Toeplitz numerical range is immediate: $W\left(T P_{A}, A\right)=W\left(\left.T A^{+}\right|_{A(H)}\right)$.

2. The convex hull of the essential spectrum. For simplicity we assume in this paragraph that all operators are bounded. There are many possible definitions of the essential spectrum of $T$; e.g., the semi-Fredholm spectrum $\sigma_{S F}(T)$, the Fredholm spectrum $\sigma_{F}(T)$, the Weyl spectrum $\omega(T)$, or the Browder spectrum. But it is easy to see that the convex hull is the same for any one of the various essential spectra and the essential radius $r_{e s s}(T)=\sup \left\{|\lambda|: \lambda \in \sigma_{e s s}(T)\right\}$ is uniquely defined.

Proposition 2.1. Let $H$ be a separable Hilbert space and $T \in \mathscr{B}(H)$. Then co $\sigma_{e s s}(T) \subset \bigcap\left\{\overline{W(A T P, A P)}: A \in \Phi_{+}, P\right.$ a projection with $\operatorname{nul}(P)<\infty\} \subset W_{\text {ess }}(T)$.

Proof. If $\lambda \notin \cap \overline{W(A T P, A P)}$, then $\lambda \notin \overline{W(A T P, A P)}$ for some $A \in \Phi_{+}$, some projection $P$ with finite nullity; i.e., there exists a $\delta>0$ such that

$$
\begin{aligned}
\delta\|A P x\| & \leqq|(A T P x, A P x)-\lambda| \\
& \leqq\|A(T-\lambda) P x\| \\
& \leqq\|A\|\|(T-\lambda) P x\| .
\end{aligned}
$$


Thus $(T-\lambda) P$ and therefore $T-\lambda \in \Phi_{+}$which yields

$$
\sigma_{S F}(T) \subset \bigcap\left\{\overline{W(A T P, A P)}: A \in \Phi_{+}, P \text { a projection with } \operatorname{nul}(P)<\infty\right\}
$$

and the first inclusion.

For the second inclusion we let $P_{n} H=\overline{s p}\left\{e_{i}: i \geqq n\right\}$ where $\left\{e_{i}: i=1,2, \cdots\right\}$ is an orthonormal basis for $H$. If $\lambda \in \bigcap \overline{W\left(T P_{n}, P_{n}\right)}$, then there exists $y_{n}$ such that $\left\|P_{n} y_{n}\right\|=1$ and $\left|\left(T P_{n} y_{n}, P_{n} y_{n}\right)-\lambda\right|<1 / n$. If $x_{n}=P_{n} y_{n}$, then $x_{n} \stackrel{w}{\rightarrow} 0,\left\|x_{n}\right\|=1$ and $\left(T x_{n}, x_{n}\right) \rightarrow \lambda$ so that $\lambda \in W_{\text {ess }}(T)$ by Theorem 5.1 of Fillmore, Stampfli, and Williams [6]. But $\cap\{\overline{W(A T P, A P}): A \in \Phi_{+}, P$ a projection with

$$
\operatorname{nul}(P)<\infty\} \subset \bigcap\left\{\overline{W\left(T P_{n}, P_{n}\right)}: n=1,2, \cdots\right\} .
$$

THEOREM 2.2. Let $H$ be a separable Hilbert space and $T \in \mathscr{B}(H)$. Then $r_{e s s}(T)=\inf \left\{\left\|A T A^{+} /_{A P(H)}\right\|: A \in \Phi_{+}, P \quad\right.$ a projection with $\left.\operatorname{nul}(P)<\infty, P_{A} P=P\right\}$.

Proof. By the remark made at the end of paragraph 1 and the fact that $A^{+} A P=P_{A} P=P$, it is clear that Proposition 2.1 and the Schwarz inequality imply that $r_{e s s}(T) \leqq \inf \left\{\left\|A T A^{+} /_{A P(H)}\right\|: A \in \Phi_{+}, P\right.$ a projection with nul $\left.(P)<\infty, P=P_{A} P\right\}$. We now prove the reverse inequality; i.e., if $r_{e s s}(T)<1$, then for any $\varepsilon>0$, there exists an $A \in \Phi_{+}, A: H \rightarrow l_{+}^{2}(H)$ and a projection $P: H \rightarrow H$ with nul $(P)<\infty$, $P_{A} P=P$ such that $\sup \{\|A T P x\|:\|A P x\|=1\} \leqq 1+\varepsilon$.

According to a theorem of Stampfli (cf. Lancaster [21]) for any $T \in \mathscr{S}(H)$, there exists a compact operator $K$ such that $\sigma(T+K)=$ $\omega(T)$. If $\varepsilon>0$ is given, there exists a projection $P: H \rightarrow H$ with nul $(P)<\infty$ such that $\|K P\|<\varepsilon / \sum_{n=0}^{\infty}\left\|(T+K)^{n}\right\|^{2}$ since $H$ is a Hilbert space and the spectral radius of $T+K, r(T+K)<1$. (We omit the trivial case $T=-K)$. If $A: H \rightarrow l_{+}^{2}(H)$ is defined by $A x=$ $\left(P x, P(T+K) x, \cdots, P(T+K)^{n} x, \cdots\right)$ then

$$
\|P x\|^{2} \leqq\|A x\|^{2} \leqq \sum_{n=0}^{\infty}\left\|(T+K)^{n}\right\|^{2}\|x\|^{2} .
$$

It is clear that $A \in \Phi_{+}$and $P_{A} P=P$. Also $A(T+K)=U_{+}^{*} A$ where $U_{+}^{*}$ is the backward shift. Thus

$$
\begin{aligned}
\|A T P x\| & \leqq\|A K P x\|+\left\|U_{+}^{*} A P x\right\| \\
& \leqq\|A\|\|K P\|\|P x\|+\left\|U_{+}^{*} A P x\right\| .
\end{aligned}
$$

Taking the sup over $\|A P x\|=1$ yields the desired result.

THeOREM 2.3. Let $T \in \mathscr{B}(H)$ where $H$ is a separable Hilbert space. Then 


$$
\begin{aligned}
\operatorname{co} \sigma_{e s s}(T)= & \left.\bigcap \overline{W(A T P, A P}): A \in \Phi_{+}, P \text { a projection with } \operatorname{nul}(P)<\infty\right\} \\
= & \bigcap\left\{\overline{W(A T P, A P)}: A \in \Phi_{+}, P \text { a projection with } \operatorname{nul}(P)<\infty,\right. \\
& \left.P_{A} P=P\right\} .
\end{aligned}
$$

Proof. In view of Proposition 2.1, we need only show

$$
\begin{aligned}
& \bigcap\{\overline{W(A T P, A P}): A \in \Phi_{+}, P \text { a projection with } \operatorname{nul}(P)<\infty, \\
& \left.P_{A} P=P\right\} \subset \cos \sigma_{e s s}(T) .
\end{aligned}
$$

The proof of this inclusion is based on the fact that any closed, convex subset of the plane is the intersection of all open discs containing it. Let $D$ be an open disc containing co $\sigma_{e s s}(T)$ with center $\lambda$ and radius $r$. Then the essential spectrum of $(1 / r)(T-\lambda I)$ lies in the open unit dise so there exists an $A \in \Phi_{+}$and a projection $P$ of finite nullity such that $\sup \|(1 / r) A(T-\lambda I) P x\|:\|A P x\|=1\}<1$. Thus $|W|((1 / r) A(T-\lambda I) P, A P)<1$ or ||$W|(A T P, A P)-\lambda|<r$; i.e., $\overline{W(A T P, A P)}$ is contained in $D$.

This proof is similar to one due to J.P. Williams in showing the Givens-Hildebrandt result $\operatorname{co} \sigma(T)=\bigcap\left\{\overline{W\left(S T S^{-1}\right.}\right): S$ is invertible $\}$; cf. Fillmore [5], p. 22. To show the analogy between the GivensHildebrandt result and our result we can rewrite the result of the theorem as co $\sigma_{e s s}(T)=\bigcap\left\{\overline{W\left(A T A^{+} /_{A P(H)}\right):} A \in \Phi_{+}, P\right.$ a projection of finite nullity\}.

3. Extensions to Banach and Frechét spaces. Theorem 1.3 can be extended to a certain extent to Banach or Frechet spaces by the use of Lumer [22] semi-inner products or "states", cf. Bonsall and Duncan [3]. E.g., if $X$ and $Y$ are Banach spaces and $T, A \in \mathscr{B}(X, Y)$, by the Hahn Banach theorem there exists a choice function $\xi: X \rightarrow X^{*}$ such that $\langle x, \xi(x)\rangle=\|x\|$ and $\|\xi(x)\|=1$. The $\operatorname{map} \zeta(x)=\|x\| \xi(x)$ then defines a semi-inner product $[\cdot, \cdot]_{\zeta}$ on $X \times X$ by

$$
\left[x_{1}, x_{2}\right]_{\zeta}=\left\langle x_{1}, \zeta\left(x_{2}\right)\right\rangle \text {. }
$$

It is easily verified that this semi-inner product satisfies most of the usual properties of an inner product except for conjugate linearity; cf. Lumer [22], Giles [7]. Since our numerical range theory does not need conjugate linearity we can define

$$
W(T, A, \zeta)=\operatorname{co}\left\{[T x, A x]_{\zeta}:\|A x\|=1\right\} \text {. }
$$

It can then be verified that Theorem 1.3 holds for $W\left(T P_{A}, A, \zeta\right)$ in place of $W\left(T P_{A}, A\right)$ for any $\zeta$ (where $P_{A}$ is the continuous projection 
taking $X$ onto $M$, the closed complement of $N(A)$ in $X)$. For a Banach space, however, equality $\gamma(A)=\gamma\left(A /_{M}\right)$ is not valid in general and therefore the remarks made immediately after Theorem 1.3 are not quite relevant to this case. Much the same can be said about operators $T, A \in L(X, Y)$ for $(X, \tau)$ and $(Y, v)$ Frechét spaces with topologies $\tau$ and $v$ generated by the families of semi-norms $\Gamma$ and $\Delta$, respectively. According to a lemma of R. T. Moore [23], for every $p \in \Delta$, there exists a choice function $\zeta_{p}: Y \rightarrow Y^{*}$ such that

$$
p(y)^{2}=\left\langle y, \zeta_{p}(y)\right\rangle
$$

for every $y \in Y$ and

$$
\left|\left\langle y_{1}, \zeta_{p}\left(y_{2}\right)\right\rangle\right| \leqq p\left(y_{1}\right) p\left(y_{2}\right) \text {. }
$$

For every $p \in \Delta$ select a $\zeta_{p}$ and define $\Lambda_{1}=\left\{\zeta_{p}: p \in \Delta\right\}$. By using techniques developed in Amelin [1], it can be shown that Theorem 1.3 holds with $W\left(T P_{A}, A\right)$ replaced by

$$
W\left(T P_{A}, A, \Lambda_{1}\right)=\operatorname{co}\left\{\left[T P_{A} x, A x\right]_{\zeta_{p}}: \zeta_{p} \in \Lambda_{1}, p(A x)=1\right\} \text {. }
$$

\section{Examples.}

(a) Let $T, A \in \mathscr{B}\left(l_{\infty}^{2}\right)$ be defined as $T=U P(0), A=U P(0) P(1)$ where $U$ is the bilateral shift, and if $e_{i}=\left\{\left(\xi_{n}\right) \mid \xi_{i}=1, \xi_{j}=0, i \neq j\right\}$, then

$$
P(j) e_{i}=\left(1-\delta_{i j}\right) e_{i} \text {. }
$$

$P_{A}=P(0) P(1)$, so $T P_{A}=A$ and

$$
W\left(T P_{A}, A\right)=\left\{\|A x\|^{2}:\|A x\|=1\right\}=\{1\} .
$$

Thus if $\lambda \neq 1$, ind $(T-\lambda A)=$ ind $(A)=0$. We note that previous results stated that for $|\lambda|>\|T\| / \gamma(A)=1$, ind $(T-\lambda A)=\operatorname{ind}(A)$.

(b) Cf. Goldberg and Schubert [15] $(G-S)$. Let $T=D^{n}$ and $A=T-\lambda$ (for $\lambda \in \boldsymbol{R}$ and $n$ odd) be the maximal operators on $L^{2}[0, \infty)$; i.e., $\mathscr{D}(T)=\mathscr{D}(A)=\left\{f: f \in L^{2}[0, \infty), f^{(n-1)}\right.$ absolutely continuous on $\left.[0, \infty), T f \in L^{2}[0, \infty)\right\}$. By Goldberg [14] Ch. 6, $A$ is Fredholm with $\operatorname{nul}(A) \leqq n$, def $(A)=0$. By Theorem 9 of $G-S, \gamma(A)=$ $\min \left\{\left|(i \sigma)^{n}-\lambda\right|: \sigma \in R\right\}=|\lambda|$. Since

$$
\|T f\| \leqq|\lambda|\|f\|+\|A f\|, \quad f \in \mathscr{D}(T),
$$

by Kato's result, if $|\xi|>(a / \gamma(A))+b=2$, then

$$
\text { ind }(T-\xi A)=\operatorname{ind}(A) \text {. }
$$

On the other hand, 


$$
\begin{aligned}
W\left(T P_{A}, A\right) & =\left\{\left(T P_{A} f, A f\right):\|A f\|=1\right\} \\
& =\left((T-\lambda) P_{A} f, A f\right)+\lambda\left(P_{A} f, A f\right) \\
& =1+\lambda\left(P_{A} f, A f\right) .
\end{aligned}
$$

But $\left|\left(P_{A} f, A f\right)\right| \leqq\left\|P_{A} f\right\| \leqq\|A f\| / \gamma(A)=1 /|\lambda| ; \quad$ i.e., $\quad \lambda\left(P_{A} f, A f\right) \subset$ $\{z:|z| \leqq 1\}$. Thus $\overline{W\left(T P_{A}, A\right)}$ is contained in a circle of radius 1 centered at $(1,0)$.

\section{REFERENCES}

1. C. F. Amelin, Norm and metric topologies, Fredholm theory, and generalized numerical range for locally convex space operators, $\mathrm{Ph}$. $\mathrm{D}$. thesis, University of California, Berkeley (1972).

2. F. V. Atkinson, The normal solubility of linear equations in normed spaces, Mat. Sb. N. S., 28 (70), 3-14 (1951).

3. F. F. Bonsall and J. Duncan, Numerical Ranges of Operators on Normed Spaces and of Elements of Normed Algebras, Lond. Math. Soc. Lecture Note Ser. (2) Cambridge Univ. Press (1971).

4. J. Dieudonné, Sur les homomorphismes d'espaces normes, Bull. Sci. Math., (2) 67 (1943), 72-84.

5. P. A. Fillmore, Notes on Operator Theory, Van Nostrand Reinhold Co., New York 1970 .

6. P. A. Fillmore, J. G. Stampfli, and J. P. Williams, On the essential numerical range, the essential spectrum and a problem of Halmos, Technical report, University of Indiana.

7. J. R. Giles, Classes of semi-inner product spaces, Trans. Amer. Math. Soc., 129 (1967), 436-446.

8. W. Givens, Field of values of a matrix, Proc. Amer. Math. Soc., 3 (1952), 206-209.

9. I. C. Gohberg, On linear equations in Hilbert space, Dokl. Akad. Nauk SSSR (N.S.), 76 (1951), 9-12.

10. - On linear equations in normed spaces, Dokl. Akad. Nauk SSSR (N.S.), 76 (1951), 477-480.

11. - On linear operators depending analytically on a parameter, Dokl. Akad. Nauk SSSR (N.S.), 78 (1951), 629-632.

12. - On the index of an unbounded operator, Mat. Sb. N. S., 33 (75) (1953), 193-198.

13. I. C. Gohberg and M. G. Krein, Fundamental theorems on deficiency numbers, root numbers, and indices of linear operators, Usp. Mat. Nauk., 12 (1957), 43-118.

14. S. Goldberg, Unbounded Linear Operators: Theory and Applications, McGrawHill, New York 1966.

15. S. Goldberg and C. Schubert, Some applications of the theory of unbounded operators to ordinary differential equations, J. Math. Anal. and Appl., 19 (1967), 78-92. 16. P. R. Halmos, A Hilbert Space Problem Book, Van Nostrand, Princeton 1967.

17. S. Hildebrandt, Uber den numerischen Wertebereich eines operators, Math. Ann., 163 (1966), 230-247.

18. T. Kato, Perturbation theory for nullity, deficiency and other quantities of linear operators, J. d'Analyse Math., 6 (1958), 273-322.

19. — Perturbation Theory for Linear Operators, Springer-Verlag, New York 1966.

20. M. G. Krein and M. A. Krasnosel'skii, Stability of the index of an unbounded operator, Mat. Sb. N. S., 30 (72) (1952), 219-224. 
21. J. S. Lancaster, Lifting from the Calkin algebra, Ph. D. thesis, Indiana University (1972).

22. G. Lumer, Semi-inner product spaces, Trans. Amer. Math. Soc., 100 (1961). 29-43.

23. R. T. Moore, Adjoints, numerical ranges and spectra of operators on locally convex spaces, Bull. Amer. Math. Soc., 75 (1969), 85-90.

24. S. M. Nikol'skii, Linear equations in linear normed spaces, Izv. Akad. Nauk SSSR. Ser. Mat., 7 (1943), 147-166.

25. Martin Schechter, Principles of Functional Analysis, Academic Press, New York 1971.

26. J. G. Stampfli and J. P. Williams, Growth conditions and the numerical range in a Banach algebra, Tôhoku Math. J., 20 (1968), 417-424.

27. M. H. Stone, Hausdorff's theorems concerning hermitian forms, Bull. Amer. Math. Soc., 36 (1930), 259-261.

28. - Linear transformations in Hilbert space, Amer. Math. Soc. Colloq. Publ.. 15 (1932).

29. B. Sz.-Nagy, On the stability of the index of unbounded linear iransformations, Acta Math. Acad. Sci. Hungar, 3 (1952), 49-52.

30. O. Toeplitz, Das algebraische analogon zu einem satze von Fejér, Math Zeit., 2 (1918), 187-197.

31. Wintner, Spektraltheorie der unendlichen matrizen, Leipzig (1930).

Received June 23, 1972 and in revised form February 28, 1973.

UNIVERSity OF MARYLAND 



\section{PACIFIC JOURNAL OF MATHEMATICS}

\section{EDITORS}

RICHARD ARENS (Managing Editor)

University of California

Los Angeles, California 90024

\author{
R. A. Beaumont \\ University of Washington \\ Seattle, Washington 98105
}

J. Dugundu*

Department of Mathematics

University of Southern California

Los Angeles, California 90007

D. Gilbarg and J. Milgram

Stanford University

Stanford, California 94305

\section{ASSOCIATE EDITORS}
E. F. BECKENBACH
B. H. NeumanN
F. WOLF
K. YosHIDA

\section{SUPPORTING INSTITUTIONS}

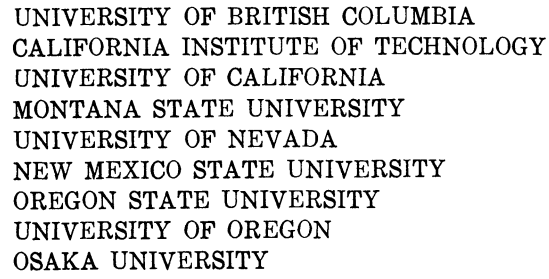

UNIVERSITY OF BRITISH COLUMBIA CALIFORNIA INSTITUTE OF TECHNOLOGY

UNIVERSITY OF CALIFORNIA

MONTANA STATE UNIVERSITY

UNIVERSITY OF NEVADA

NEW MEXICO STATE UNIVERSITY

OREGON STATE UNIVERSITY

UNIVERSITY OF OREGON

OSAKA UNIVERSITY

\author{
UNIVERSITY OF SOUTHERN CALIFORNIA \\ STANFORD UNIVERSITY \\ UNIVERSITY OF TOKYO \\ UNIVERSITY OF UTAH \\ WASHINGTON STATE UNIVERSITY \\ UNIVERSITY OF WASHINGTON \\ * * * \\ AMERICAN MATHEMATICAL SOCIETY \\ NAVAL WEAPONS CENTER
}

The Supporting Institutions listed above contribute to the cost of publication of this Journal, but they are not owners or publishers and have no responsibility for its content or policies.

Mathematical papers intended for publication in the Pacific Journal of Mathematics should be in typed form or offset-reproduced, (not dittoed), double spaced with large margins. Underline Greek letters in red, German in green, and script in blue. The first paragraph or two must be capable of being used separately as a synopsis of the entire paper. Items of the bibliography should not be cited there unless absolutely necessary, in which case they must be identified by author and Journal, rather than by item number. Manuscripts, in duplicate if possible, may be sent to any one of the four editors. Please classify according to the scheme of Math. Rev. Index to Vol. 39. All other communications to the editors should be addressed to the managing editor, or Elaine Barth, University of California, Los Angeles, California, 90024.

50 reprints are provided free for each article; additional copies may be obtained at cost in multiples of 50 .

The Pacific Journal of Mathematics is issued monthly as of January 1966. Regular subscription rate: $\$ 48.00$ a year (6 Vols., 12 issues). Special rate: $\$ 24.00$ a year to individual members of supporting institutions.

Subscriptions, orders for back numbers, and changes of address should be sent to Pacific Journal of Mathematics, 103 Highland Boulevard, Berkeley, California, 94708.

PUBLISHED BY PACIFIC JOURNAL OF MATHEMATICS, A NON-PROFIT CORPORATION

Printed at Kokusai Bunken Insatsusha (International Academic Printing Co., Ltd.), 270, 3-chome Totsuka-cho. Shinjuku-ku, Tokyo 160, Japan.

* C. R. DePrima California Institute of Technology, Pasadena, CA 91109, will replace J. Dugundji until August 1974.

Copyright (C) 1973 by

Pacific Journal of Mathematics

All Rights Reserved 


\section{Pacific Journal of Mathematics}

Vol. 48, No. $2 \quad$ April, 1973

Mir Maswood Ali, Content of the frustum of a simplex................

Mieczyslaw Altman, Contractors, approximate identities and factorization

in Banach algebras ................................ 323

Charles Francis Amelin, A numerical range for two linear operators ...... 335

John Robert Baxter and Rafael Van Severen Chacon, Nonlinear functionals

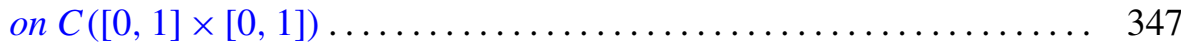

Stephen Dale Bronn, Cotorsion theories....................... 355

Peter A. Fowler, Capacity theory in Banach spaces............... 365

Jerome A. Goldstein, Groups of isometries on Orlicz spaces ........... 387

Kenneth R. Goodearl, Idealizers and nonsingular rings . ............ 395

Robert L. Griess, Jr., Automorphisms of extra special groups and

nonvanishing degree 2 cohomology ..................... 403

Paul M. Krajkiewicz, The Picard theorem for multianalytic functions . . . . 423

Peter A. McCoy, Value distribution of linear combinations of axisymmetric harmonic polynomials and their derivatives ...................

A. P. Morse and Donald Chesley Pfaff, Separative relations for

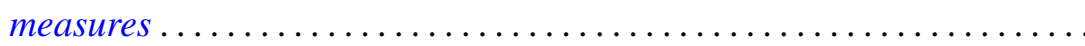

Albert David Polimeni, Groups in which $\operatorname{Aut}(G)$ is transitive on the

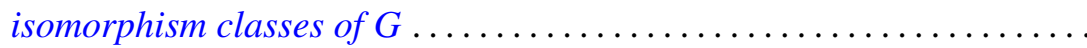

Aribindi Satyanarayan Rao, Matrix summability of a class of derived

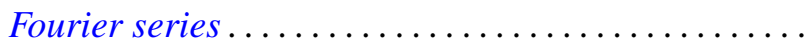

Thomas Jay Sanders, Shape groups and products

Ruth Silverman, Decomposition of plane convex sets. II. Sets associated

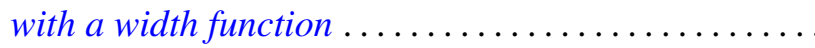

Richard Snay, Decompositions of $E^{3}$ into points and countably many flexible dendrites.............................

John Griggs Thompson, Nonsolvable finite groups all of whose local subgroups are solvable, IV ...

Robert E. Waterman, Invariant subspaces, similarity and isometric equivalence of certain commuting operators in $L_{p} \ldots$

James Chin-Sze Wong, An ergodic property of locally compact amenable

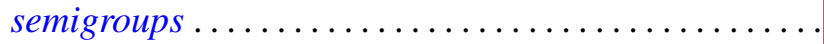

Julius Martin Zelmanowitz, Orders in simple Artinian rings are strongly equivalent to matrix rings ....................... 